\title{
A major Ku?
}

Since its discovery, $\underline{B C L 2}$ has been viewed as a mild-mannered oncogene - tumorigenic over a protracted time course in comparison with seasoned professionals such as $\underline{M Y C}$ and KRAS. However, over recent years evidence has been emerging that BCL2 might have a more sinister function: that it might be involved in inhibiting DNA repair.

Xingming Deng and colleagues have evidence that BCL2 can block the function of the DNA repair complex Ku. Ku exists as a KU70 (also known as XRCC6)-KU80 (also known as XRCC5) heterodimer that binds the ends of DNA double-strand breaks (DSBs). By recruiting the catalytic subunit of DNA-protein kinase (DNA-PKcs, also known as PRKDC) and other proteins, Ku enables the repair of DNA DSBs through the non-homologous end joining pathway.

The authors initially observed that lung cancer cells with increased expression of endogenous BCL2 have reduced Ku end-binding capacity. Overexpression of exogenous BCL2 in a lung cancer cell line that expresses no detectable level of the endogenous protein resulted in decreased DNA DSB repair, as shown by persistent DNA DSBs (shown using pulsed field gel electrophoresis) and $\gamma \mathrm{H} 2 \mathrm{AX}$ foci, and increased levels of cytogenetic abnormalities compared with controls after exposure to ionizing radiation (IR). Furthermore, they showed that endogenous BCL2 accumulates in the nucleus after IR and that nuclear BCL2 can co-immunoprecipitate KU70.
Expression of deletion mutants of BCL2 indicates that BCL2 homology (BH) domains 1 and 4 are required for this interaction, and that this function of BCL2 seems to be independent of its anti-apoptotic role. Moreover, it seems that binding of BCL2 to Ku prevents the interaction of Ku with DNA ends and with DNAPKcs, and so decreases the activity of the DNA-PK complex.

Although on the surface and in terms of oncogenesis these findings make sense, it seems counter-intuitive that a protein capable of increasing cell survival after exposure to DNA-damaging agents would also have the capacity to prevent the repair of DNA DSBs. Perhaps then, in light of these findings, we should return to the mouse models of BCL2 overexpression and take a closer look at how BCL2 can promote tumorigenesis in vivo.

Nicola McCarthy

ORIGINAL RESEARCH PAPER Wang, Q. et al.

$B c 12$ negatively regulates DNA double strand break repair through non-homologous endjoining pathway. Mol. Cell 29, 488-498 (2008) FURTHER READING Letai, A. Diagnosing and exploiting cancer's addiction to blocks in apoptosis. Nature Rev. Cancer 8, 121-132 (2008)

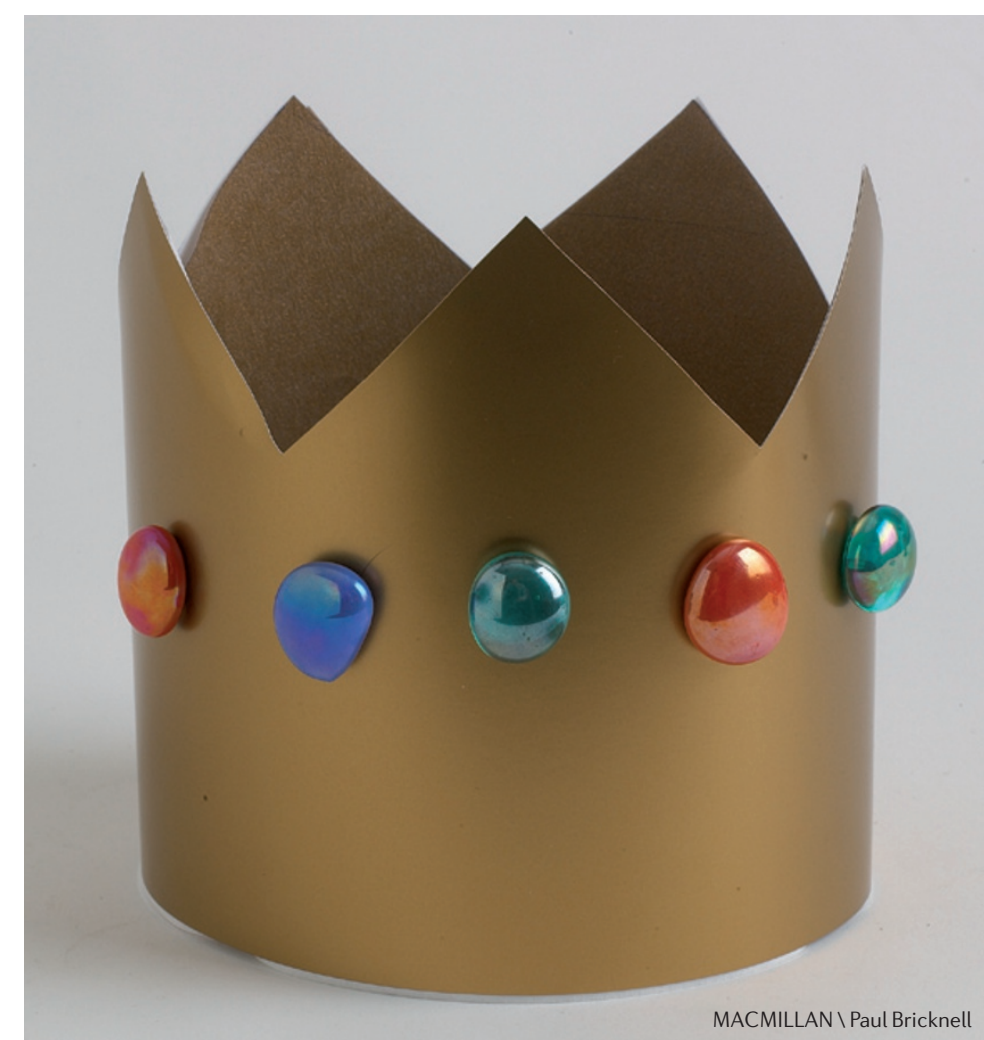

